\title{
Smaller protein, larger therapeutic potential: PPM1D as a new therapeutic target in brainstem glioma
}

"The epidemiology of PPM1D alterations in all types of cancer - which is still being uncovered - will illuminate the function of the mutations and guide therapeutic development work."

Keywords: diffuse intrinsic pontine glioma $\bullet$ protein phosphatase 1D • wild-type p53 induced phosphatase $1 \bullet$ WIP1

\section{Gain-of-function PPM1D mutations in brainstem gliomas}

Brainstem gliomas are a heterogeneous group of tumors of the midbrain, pons, medulla and/or cervical spine. One of the most frequent types of brainstem gliomas is the diffuse intrinsic pontine glioma, a devastating childhood tumor [1]. Genome-wide analyses aimed at understanding brainstem glioma biology, stratifying patients for clinical trials and discovering therapeutic targets have recently culminated with five high-profile reports [2-6]. A total of $9-23 \%$ of diffuse intrinsic pontine gliomas were found to harbor heterozygous, somatic mutations in PPM1D (also called WIP1) [2-4]. This discovery provides a potential therapeutic target for a deadly brain tumor, and adds a new dimension to the complex role for the PPMID proto-oncogene in human cancer.

\section{Normal function of PPM1D}

PPM1D dephosphorylates multiple DNA damage response (DDR) components, including p53, Chk2, ATM and $\gamma-\mathrm{H} 2 \mathrm{Ax}$. By doing so, it antagonizes these checkpoint proteins to promote re-entry into the cell cycle following activation of the DDR. Inappropriate activation of PPM1D in vitro has been linked to transformation and impairment of apoptosis by suppressing downstream DDR modulators [7]. The genetic landscape of brainstem gliomas makes it clear that PPM1D mutations disrupt the DDR axis: mutations of PPM1D and TP53, and rare mutations of $A T M$ and $C H E K 2$, occur in a nonoverlapping fashion $[2,4,5]$.

\section{Cancer-derived mutations truncate \& stabilize PPM1D}

The PPMID mutations in brainstem gliomas truncate PPM1D to express a shorter, more stable PPM1D protein. Most mutations are nonsense mutations clustered in exon 6 around codons 460-560 [2]. These mutations leave the phosphatase domain (residues 1-370) intact and functional, but remove a C-terminal tail that probably regulates turnover of the protein $[8,9]$. The truncated protein has a longer half-life in cells, and the persistent mutant PPM1D protein promotes dephosphorylation of $\mathrm{p} 53$, Chk 2 and $\gamma-\mathrm{H} 2 \mathrm{Ax} \quad[2,9-11]$. The C-terminal tail of PPM1D contains numerous functional motifs [8], raising the possibility that truncating mutations could have additional effects on the function or localization of PPM1D.

\section{A constellation of PPM1D alterations in cancer}

The epidemiology of PPM1D alterations in all types of cancer - which is still being uncovered - will illuminate the function of the mutations and guide therapeutic development work.

Mutations in nonbrainstem tumors

Somatic, truncating PPM1D mutations have been found at low frequencies in glio-

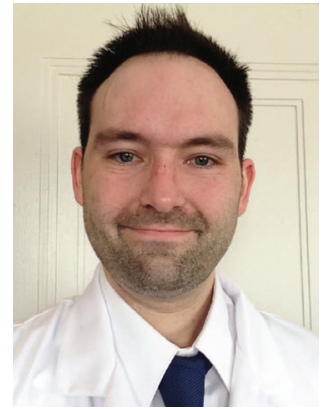

Zachary J Reitman Department of Internal Medicine, Medstar Union Memorial Hospital, Baltimore, MD, USA Tel.: +1 4104670593 zach.reitman@gmail.com 
mas outside the brainstem $[2,3]$, in uterine endometrioid carcinomas and a handful of other tumors outside the CNS [10], and in a colon adenocarcinoma and an osteosarcoma cell line [9]. These scattered mutations may be the 'tip of the iceberg', and additional tumor types with frequent PPMID mutations may be found with focused resequencing efforts. Somatic missense mutations that inactivate PPM1D have also been observed in several cancers [10], but it is unclear whether these rare inactivating mutations play a biological role in cancer.

"This puzzling finding of an allele that predisposes to cancer yet disappears in the tumor itself warrants further study.

\section{Germline mutations}

PPM1D mutations have been discovered in germline tissues and in a germline mosaic distribution in cancer patients. The germline PPM1D mutations - also truncating, activating mutations - were present in peripheral blood collected from approximately $1 \%$ of patients with colorectal and breast cancers, implicating PPMID as a cancer predisposition gene [9,1]. Surprisingly, the PPM1D mutations found in those patients' germlines were not always present in their tumors. This puzzling finding of an allele that predisposes to cancer yet disappears in the tumor itself warrants further study.

\section{Amplification}

PPMID was first established as a proto-oncogene over a decade ago after it was found to be a target of copy number amplification and overexpression in breast and other tumor types [7]. One brainstem glioma harbored a PPM1D amplification in our study [2], indicating that amplification and truncating mutations have overlapping oncogenic functions.

\section{Splicing variant}

A natural alternative splicing variant of PPM1D, termed PPM1D430, is only expressed in testes and leukocytes [12]. PPM1D430 comprises the first 420 residues common to full-length PPM1D, including the entire phosphatase domain and a unique ten C-terminal residues - a very similar configuration to cancer-derived truncated PPM1D. Understanding the physiological role of PPM1D430 in these proliferative tissues may provide clues about the role of truncated PPM1D mutants in tumor cells.

\section{Mutant PPM1D as a therapeutic target}

Early studies implicate mutant PPM1D as a therapeutic target, and experimental small molecules targeting it could already be available.
Mutant PPM1D: an oncogenic driver

Truncated PPM1D mutants oppose $\gamma$-H2Ax, p53 and Chk2 activation in response to ionizing radiation [2,9-11]. This finding indicates that the mutations drive tumor growth by opposing the DDR and allowing tumor cells to progress through the cell cycle and avoid apoptosis. We found that removing the PPMID mutation found in a colon adenocarcinoma cell line by genetic recombination led to slowed cell line growth - indicating that PPM1D-mutated cancer cells depend on mutant PPM1D function for continued proliferation [2]. Together, these findings point to inhibition of mutant PPM1D as a therapeutic strategy. Genetically engineered mice completely lacking PPMID are viable into adulthood (although with reproductive and immune system defects), which bodes well for the side-effect profile of any therapy targeting PPM1D [13,14].

\section{Existing PPM1D inhibitors}

The discovery of oncogenic PPM1D amplifications in breast cancer spurred the development of small molecule PPM1D inhibitors, which predate the discovery of the PPMID mutations in brainstem gliomas. Molecules reported to date include a cyclic phosphopeptide inhibitor [15], a compound identified in a screen that specifically kills PPM1D-overexpressing cell lines [16,17], a unique uncompetitive inhibitor [18], and an orally active small molecule which allosterically inhibits PPM1D and which showed inhibition of a mouse lymphoma tumor graft model with diploid PPM1D [19]. Since they target the phosphatase domain of PPM1D, these small molecules can probably inhibit truncated mutants of PPM1D found in brainstem gliomas and could act as prototypes for new therapies.

\section{Future directions}

The discovery of PPMID mutations in brainstem gliomas opens the door to promising clinical and therapeutic studies.

\section{Clinical correlates \& tumor heterogeneity}

Follow-up of a larger cohort of brainstem glioma patients will be needed to determine whether PPMID mutations associate with different baseline clinical characteristics or different median survival. Also, an open question remains as to whether PPM1D mutation is present throughout brainstem glioma tissues or only in subclonal populations, and whether the mutation is stable after tumor progression. These questions must be resolved by analysis of microdissected and recurrent tumors to help prioritize PPMID as a therapeutic target. 


\section{Preclinical studies}

It remains to be formally confirmed that compounds designed to inhibit full-length PPM1D can inhibit truncated PPM1D mutants. Also, it is critical to develop PPM1D-mutated brainstem glioma models, including genetically engineered animals that express mutated PPM1D in tumors, and animals with tumor grafts derived from PPM1D-mutated human gliomas. These models will enable testing whether drugs targeting PPM1D can cure PPM1D-mutated brainstem gliomas in combination with radiotherapy or other drugs. Nonglioma cell lines that harbor PPM1D mutations represent important tools that are already available to interrogate the function of mutant PPM1D [9].

\section{Future clinical trials?}

Brainstem gliomas present several challenges for testing investigational new drugs. The tumors are rare,

\section{References}

1 Hargrave D, Bartels U, Bouffet E. Diffuse brainstem glioma in children: critical review of clinical trials. Lancet Oncol. 7(3), 241-248 (2006).

2 Zhang $\mathrm{L}$, Chen LH, Wan $\mathrm{H}$ et al. Exome sequencing identifies somatic gain-of-function PPM1D mutations in brainstem gliomas. Nat. Genet. 46(7), 726-730 (2014).

3 Wu G, Diaz AK, Paugh BS et al. The genomic landscape of diffuse intrinsic pontine glioma and pediatric non-brainstem high-grade glioma. Nat. Genet. 46(5), 444-450 (2014).

4 Taylor KR, Mackay A, Truffaux N et al. Recurrent activating ACVR1 mutations in diffuse intrinsic pontine glioma. Nat. Genet. 46(5), 457-461 (2014).

5 Fontebasso AM, Papillon-Cavanagh S, Schwartzentruber J et al. Recurrent somatic mutations in ACVR1 in pediatric midline high-grade astrocytoma. Nat. Genet. 46(5), 462-466 (2014).

6 Buczkowicz P, Hoeman C, Rakopoulos P et al. Genomic analysis of diffuse intrinsic pontine gliomas identifies three molecular subgroups and recurrent activating $A C V R 1$ mutations. Nat. Genet. 46(5), 451-456 (2014).

7 Li J, Yang Y, Peng Y et al. Oncogenic properties of PPM1D located within a breast cancer amplification epicenter at 17q23. Nat. Genet. 31(2), 133-134 (2002).

8 Yoda A, Xu XZ, Onishi $\mathrm{N}$ et al. Intrinsic kinase activity and $\mathrm{SQ} / \mathrm{TQ}$ domain of Chk2 kinase as well as $\mathrm{N}$-terminal domain of Wip1 phosphatase are required for regulation of Chk2 by Wip1. J. Biol. Chem. 281(34), 24847-24862 (2006).

9 Kleiblova P, Shaltiel IA, Benada J et al. Gain-of-function mutations of PPM1D/Wip1 impair the p53-dependent G1 checkpoint. J. Cell. Biol. 201(4), 511-521 (2013).

10 Dudgeon C, Shreeram S, Tanoue K et al. Genetic variants and mutations of PPM1D control the response to DNA damage. Cell Cycle 12(16), 2656-2664 (2013). and CNS drug availability must be considered. If an investigational new drug targeting PPM1D is developed, other cancer types may provide a more ideal setting for early clinical trials. Thus, focused resequencing to determine whether other tumor types harbor frequent PPM1D mutations is the first step to prioritize (and encourage) preclinical work.

\section{Financial \& competing interests disclosure}

The author has no relevant affiliations or financial involvement with any organization or entity with a financial interest in or financial conflict with the subject matter or materials discussed in the manuscript. This includes employment, consultancies, honoraria, stock ownership or options, expert testimony, grants or patents received or pending, or royalties.

No writing assistance was utilized in the production of this manuscript.

11 Ruark E, Snape K, Humburg P et al. Mosaic PPM1D mutations are associated with predisposition to breast and ovarian cancer. Nature 493(7432), 406-410 (2013).

12 Chuman Y, Kurihashi W, Mizukami Y, Nashimoto T, Yagi $\mathrm{H}$, Sakaguchi K. PPM1D430, a novel alternative splicing variant of the human PPM1D, can dephosphorylate $\mathrm{p} 53$ and exhibits specific tissue expression. J. Biochem. 145(1), 1-12 (2009).

13 Bulavin DV, Phillips C, Nannenga B et al. Inactivation of the Wip1 phosphatase inhibits mammary tumorigenesis through p38 MAPK-mediated activation of the p16(Ink4a)-p19(Arf) pathway. Nat. Genet. 36(4), 343-350 (2004).

14 Choi J, Nannenga B, Demidov ON et al. Mice deficient for the wild-type p53-induced phosphatase gene (Wip1) exhibit defects in reproductive organs, immune function, and cell cycle control. Mol. Cell. Biol. 22(4), 1094-1105 (2002).

15 Yamaguchi H, Durell SR, Feng H, Bai Y, Anderson CW, Appella E. Development of a substrate-based cyclic phosphopeptide inhibitor of protein phosphatase 2Cdelta, Wip1. Biochemistry 45(44), 13193-13202 (2006).

16 Rayter S, Elliott R, Travers J et al. A chemical inhibitor of PPM1D that selectively kills cells overexpressing PPM1D. Oncogene 27(8), 1036-1044 (2008).

17 Tan DS, Lambros MB, Rayter $S$ et al. PPM1D is a potential therapeutic target in ovarian clear cell carcinomas. Clin. Cancer Res. 15(7), 2269-2280 (2009).

18 Chuman Y, Yagi H, Fukuda T et al. Characterization of the active site and a unique uncompetitive inhibitor of the PPM1-type protein phosphatase PPM1D. Protein Pept. Lett. 15(9), 938-948 (2008).

19 Gilmartin AG, Faitg TH, Richter M et al. Allosteric Wip1 phosphatase inhibition through flap-subdomain interaction. Nat. Chem. Biol. 10(3), 181-187 (2014). 